\title{
Status of charmed meson spectroscopy
}

\author{
Feng-Kun $\mathrm{Guo}^{1,2, *}$ \\ ${ }^{1}$ CAS Key Laboratory of Theoretical Physics, Institute of Theoretical Physics, Chinese Academy of \\ Sciences, Beijing 100190, China \\ ${ }^{2}$ School of Physical Sciences, University of Chinese Academy of Sciences, Beijing 100049, China
}

\begin{abstract}
The discovery of the ground state positive-parity charm-strange and charm-nonstrange mesons $D_{s 0}^{*}(2317), D_{s 1}(2460), D_{0}^{*}(2400)$ and $D_{1}(2430)$ in 2003 and 2004 brought up several mysteries related to their masses. Here I briefly review recent progresses from lattice calculations and analysis of the precise LHCb measurements of the $B^{-} \rightarrow D^{+} \pi^{-} \pi^{-}$in the framework of unitarized chiral perturbation theory. It turns out that all the mysteries can be understood in a picture consistent with both lattice results and the LHCb measurements. In this picture, the main components of $D_{s 0}^{*}(2317)$ and $D_{s 1}(2460)$ are $D K$ and $D^{*} K$ hadronic molecules, respectively. Furthermore, the resonance parameters of the ground state $0^{+}$and $1^{+}$charm-nonstrange mesons take values very different from the known ones of the $D_{0}^{*}(2400)$ and $D_{1}(2430)$, which were obtained by using an improper resonance parameterization. It is pointed out that there should be two $D_{0}^{*}$ and two $D_{1}$ broad states in region relevant to the $D_{0}^{*}(2400)$ and $D_{1}(2430)$. Suggestions towards identifying the higher nonstrange resonances are given.
\end{abstract}

\section{Introduction}

The discovery of the $D_{s 0}^{*}(2317)$ with $J^{P}=0^{+}$by the BaBar Collaboration in 2003 [1] marked the start of a new era in the study of hadron spectroscopy. After that, tens of hadronic resonant structures were reported in experiments with properties at odds with the quark model which describes mesons as bound states of a quark and an antiquark. Many of these new hadrons are then natural candidates of exotic hadrons. For recent reviews of the experimental observations and the efforts devoted to understanding them, see Refs. [2-11]. Here I will discuss the positive-parity charmed mesons, i.e., $D_{s 0}^{*}(2317), D_{s 1}(2460), D_{0}^{*}(2400)$ and $D_{1}(2430)$.

The discovery of the $D_{s 0}^{*}(2317)$ was closely followed by that of the axial-vector meson $D_{s 1}(2460)$ by the CLEO Collaboration [12]. The masses of these two charm-strange mesons, $(2317.7 \pm 0.6) \mathrm{MeV}$ and $(2459.5 \pm 0.6) \mathrm{MeV}$, respectively [13], are much lower than the values predicted in potential quark models such as the Godfrey-Isgur (GI) quark model [14] (the updated results for charmed mesons can be found in [15]), which was very successful in describing the observed hadron spectroscopy till then. No isospin partners were found. Their widths are tiny, and so far only upper limits were reported [13]. So these two charm-strange mesons are isospin-scalar states. As they are located below the $D K$ and $D^{*} K$ thresholds, respectively, the only allowed strong decays are into $D_{s} \pi$ and $D_{s}^{*} \pi$ for the $D_{s 0}^{*}(2317)$ and

\footnotetext{
*e-mail: fkguo@itp.ac.cn
} 
$D_{s 1}(2460)$, respectively, breaking isospin symmetry. These two states spurred lots of interest because of the unexpected low masses. Attempts were made trying to explain them as $c \bar{s}$ mesons [16-20], chiral partners of the ground state $D_{s}$ and $D_{s}^{*}$ mesons [21, 22], compact [cq] $[\bar{s} \bar{q}]$ tetraquark states [23], mixing of the $c \bar{s}$ and tetraquarks [24], the $D \pi$ atom for the $D_{s 0}^{*}(2317)$ [25], and the $D^{(*)} K$ hadronic molecules [26-31].
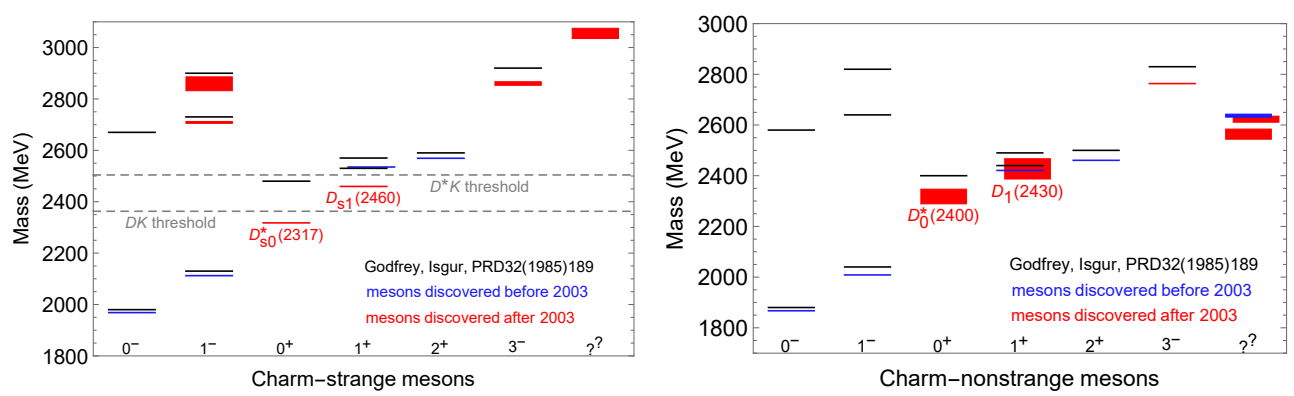

Figure 1. The charm-strange and charm-nonstrange meson mass spectra. The predictions in the GI quark model [14] are shown as in black lines, while the mesons observed before and since 2003 are shown as blue and red lines, respectively. For the observed mesons, the masses given in the RPP [13] are taken, and the bands correspond to their uncertainties.

Shortly after these discoveries, the lowest scalar and axial-vector charm-nonstrange mesons $D_{0}^{*}(2400)^{0}$ and $D_{1}(2430)^{0}$ were reported by the Belle Collaboration [32]. They show up as broad bumps in the $D^{(*)} \pi$ invariant mass distributions, and each of them was fitted with a single Breit-Wigner resonance. The $D_{0}^{*}(2400)^{0}$ was later on also reported by the FOCUS, $\mathrm{BaBar}$ and LHCb Collaborations. While all experiments reported a large width, with values consistent with each another, the central values of the mass differ largely: (2308 \pm 36$) \mathrm{MeV}$ (Belle) [32], (2407 \pm 41$) \mathrm{MeV}$ (FOCUS) [33], and (2297 \pm 22) MeV (BaBar) [34]. The averaged values is $(2318 \pm 27) \mathrm{MeV}$ in the Review of Particle Physics (RPP) [13]. The charged partner $D_{0}^{*}(2400)^{ \pm}$was reported by the FOCUS [33] and LHCb [35, 36] Collaborations, and the averaged mass is $(2351 \pm 7) \mathrm{MeV}$ [13]. One should not regard the difference between the neutral and charged RPP masses as a large isospin splitting. The mass spectra of the charmstrange and charm-nonstrange mesons are shown in Fig. 1, where the predictions in the GI quark model [14] are also shown.

These observations led to three mysteries regarding the masses of the positive-parity charmed mesons:

(1) Mass problem: Why are the $D_{s 0}^{*}(2317)$ and $D_{s 1}(2460)$ much lighter than the quark model expectations for the lowest scalar and axial-vector $c \bar{s}$ mesons?

(2) Fine-tuning problem: Why is $M_{D_{s 1}(2460)}-M_{D_{s 0}^{*}(2317)} \simeq M_{D^{* \pm}}-M_{D^{ \pm}}$within $2 \mathrm{MeV}$ ? Notice that the positive-parity states and the negative-parity ones are in different spin multiplets. If they are not connected by any symmetry, it would be mysterious why the hyperfine splittings should be fine tuned to be almost equal. In Refs. [21, 22], this equality is obtained as a consequence of the chiral-partner assumption. From Fig. 1, one sees that the splittings are very different in the GI quark model.

(3) Hierachy problem: Why are $M_{D_{0}^{*}(2400)} \gtrsim M_{D_{s 0}^{*}(2317)}$ and $M_{D_{1}(2430)} \sim M_{D_{s 1}(2460)}$ ? Such relationships were used as inputs in many works, see e.g. [19, 37-39]. But the natural mass hierachy within a given SU(3) flavor multiplet should be that the hadron with a strange quark is at least $100 \mathrm{MeV}$ heavier than the nonstrange ones, as a consequence of the strange quark being much heavier than the up and down quarks. 
Table 1. Masses of the ground state $0^{+}$and $0^{-}$charm-strange mesons in the lattice calculation using two different pion masses in Ref. [44]. The experimental values [13] are given in the last column. All values are in units of $\mathrm{MeV}$.

\begin{tabular}{llll}
\hline & $M_{\pi}=150 \mathrm{MeV}$ & $M_{\pi}=290 \mathrm{MeV}$ & $\mathrm{RPP}$ \\
\hline$M_{D_{s}}$ & $1977 \pm 1$ & $1980 \pm 1$ & $1968.34 \pm 0.07$ \\
$M_{D_{s}^{*}}$ & $2094 \pm 1$ & $2101 \pm 1$ & $2112.2 \pm 0.4$ \\
$M_{D_{s 0}^{*}}$ & $2348 \pm 4$ & $2384 \pm 3$ & $2317.7 \pm 0.6$ \\
$M_{D_{s 1}}$ & $2351 \pm 4$ & $2397 \pm 4$ & $2459.5 \pm 0.6$ \\
\hline
\end{tabular}

Although their bottom cousins have not been discovered so far, it is natural to ask whether such mysteries will repeat there.

Next I will discuss the progress on understanding these mysteries using lattice quantum chromodynamics (QCD) and effective field theory calculations.

\section{Lattice QCD studies of the positive-parity charmed mesons}

The calculation of the positive-parity charmed meson spectrum on lattice is difficult as their masses are close to the strongly-coupled $S$-wave $D^{(*)} K$ thresholds, and thus it is not easy to get the correct masses without taking the $D^{(*)} K$ into account. The inclusion of the latter, however, requires the calculation of quark-antiquark annihilation-type Wick contractions, which remained a challanging problem for a long time.

Attempts of calculating the ground state scalar charm-strange meson were made shortly after the $D_{s 0}^{*}(2317)$ discovery. Interpolating operators of the $c \bar{s}$ type were used, and the obtained masses $[40,41]$ were larger than the observed value for the $D_{s 0}^{*}(2317)$. The first calculation using interpolating operators of both the $c \bar{s}$ and $D K$ (constructed with two quark fields and two antiquark fields) types was made in $[42,43]$. The obtained value for the mass difference $M_{D_{s 0}^{*}}-\left(M_{D_{s}}+3 M_{D_{s}^{*}}\right) / 3,(266 \pm 16) \mathrm{MeV}$, was consistent with the experimental value $(241.5 \pm 0.8) \mathrm{MeV}$. A more complete lattice calculation on the charm-strange meson spectrum was made in Ref. [44], where the masses of the $0^{+}$and $1^{+}$charm-strange mesons were 2348(4) $\mathrm{MeV}$ and 2451(4) MeV, respectively, at a pion mass of $150 \mathrm{MeV}$. These values are close to the measured masses of the $D_{s 0}^{*}(2317)$ and $D_{s 1}(2460)$. The masses of the ground state $0^{-}, 1^{-}, 0^{+}$and $1^{+}$charm-strange mesons obtained in Ref. [44] using two different pion masses are shown in Table 1. It is clear that the pion-mass dependence of the $0^{+}$and $1^{+}$states is much stronger than that of the $0^{-}$and $1^{-}$ones, indicating the important role of up and down quarks in the charm-strange mesons.

The charmed-meson-light-meson scattering lengths in a few channels ( $D \pi$ with $I=3 / 2$, $D \bar{K}$ with $I=0$ and $1, D_{s} K$ and $D_{s} \pi$ ) free of quark-annihilation contractions were first calculated on lattice in Ref. [45] (updated in Ref. [46]). These results provide valuable information on the low-energy interaction between a charmed meson and a light pseudoscalar meson, and thus leads to important insights into the nature of the scalar charmed mesons, to be discussed in the next section.

As for the non-strange sector, the $D \pi I=1 / 2 S$-wave cattering length and the lowest scalar $D_{0}^{*}$ meson were calculated using the Lüscher's method in Ref. [47] using the $c \bar{s}+D \pi$ type interpolating operators. With a pion mass of about $266 \mathrm{MeV}$, the authors got $M_{D_{0}^{*}}-$ $\left(M_{D}+3 M_{D^{*}}\right) / 3=(351 \pm 21) \mathrm{MeV}$ and $M_{D_{1}}-\left(M_{D}+3 M_{D^{*}}\right) / 3=(380 \pm 21) \mathrm{MeV}$, which may be confronted with $(347 \pm 29) \mathrm{MeV}$ and $(456 \pm 40) \mathrm{MeV}$, respectively, using the masses of the $D_{0}^{*}(2400)$ and $D_{1}(2430)$ in the RPP [13].

The most updated and sophisticated lattice calculation of the $I=1 / 2 D_{0}^{*}$ is presented in Ref. [48] using the coupled-channel Lüscher's method. The $c \bar{s}+D \pi+D \eta+D_{s} \bar{K}$-type 
Table 2. Predicted masses of the $0^{+}$and $1^{+}$heavy-strange mesons, and poles given as $(M, \Gamma / 2)$ for the

$0^{+}$and $1^{+}$heavy-nonstrange mesons. Here $M$ and $\Gamma$ are the mass and the total decay width, respectively. For comparison, the RPP values [13] and latest lattice QCD results are also shown. All values are in units of $\mathrm{MeV}$.

\begin{tabular}{|c|c|c|c|}
\hline & Prediction & RPP & Lattice QCD \\
\hline$D_{s 0}^{*}$ & $2315_{-28}^{+18}$ & $2317.7 \pm 0.6$ & $2348_{-4}^{+7}[44]$ \\
\hline$D_{s 1}$ & $2456_{-21}^{+15}$ & $2459.5 \pm 0.6$ & $2451 \pm 4[44]$ \\
\hline$B_{s 0}^{*}$ & $5720_{-23}^{+16}$ & - & $5711 \pm 23[64]$ \\
\hline$B_{s 1}$ & $5772_{-21}^{+15}$ & - & $5750 \pm 25[64]$ \\
\hline$D_{0}^{*}$ & $\left(2105_{-8}^{+6}, 102_{-11}^{+10}\right), \quad\left(2451_{-26}^{+35}, 134_{-8}^{+7}\right)$ & $(2318 \pm 29,134 \pm 20)$ & - \\
\hline$D_{1}$ & $\left(2247_{-6}^{+5}, 107_{-10}^{+11}\right), \quad\left(2555_{-30}^{+47}, 203_{-9}^{+8}\right)$ & $\left(2427 \pm 40,192_{-55}^{+65}\right)$ & - \\
\hline$B_{0}^{*}$ & $\left(5535_{-11}^{+9}, 113_{-17}^{+15}\right),\left(5852_{-19}^{+16}, 36 \pm 5\right)$ & - & - \\
\hline$B_{1}$ & $\left(5584_{-11}^{+9}, 119_{-17}^{+14}\right),\left(5912_{-18}^{+15}, 42_{-4}^{+5}\right)$ & - & - \\
\hline
\end{tabular}

interpolating fields are employed, and the computed energy levels cover the energy region up to above the $D_{s} \bar{K}$ threshold. The obtained energy levels were analyzed using a coupledchannel $K$-matrix formalism. Many $K$-matrix parameterizations were used to fit the energy levels. The obtained $K$-matrix were then substituted into the infinite-volume $T$-matrix, which has poles corresponding to hadronic resonances. In this way, a pole at $(2275.9 \pm 0.9) \mathrm{MeV}$, slightly below the $D \pi$ threshold at $(2276.4 \pm 0.9) \mathrm{MeV}$, were found for $M_{\pi} \simeq 391 \mathrm{MeV}$. It is worthwhile to notice that this mass is lower than the $D_{0}^{*}(2400)$ in RPP [13], even though the pion mass takes a value much higher than its physical value.

\section{Insights from SU(3) and chiral symmetry}

The scalar (axial-vector) charmed mesons can be studied via the $S$-wave scattering between the ground state pseudoscalar (vector) charmed mesons and the light pseudoscalar mesons, and they appear as poles in the pertinent scattering amplitudes. This is the method taken in Refs. [29-31, 46, 49-58], where various versions of unitarized chiral perturbation theory (UCHPT) [59-61] were used. In short, the charmed mesons can be treated as matter fields in chiral effective Lagrangians, and the corresponding scattering amplitudes are then resummed in the $s$-channel to satisfy unitarity and to generate poles. The SU(3) flavor symmetry is incorporated into the Lagrangians, which gives the method a predictive power connecting different isospin-strangeness channels. It is instructive to notice that the flavor symmetry structure for mesonic resonances generated from such meson-meson interactions is different from that in quark models, see, e.g., [62]. As for the charmed mesons, while $c \bar{q}$ mesons form an SU(3) antitriplet ( $\overline{\mathbf{3}})$, the charmed-meson and light-meson pairs form an $\overline{\mathbf{3}}$, a $\mathbf{6}$ and a $\mathbf{1 5 .}$ The leading order chiral interactions in both the $\overline{\mathbf{3}}$ and $\mathbf{6}$ are attractive, with the strength of the former being larger. It turns out that the $D_{s 0}^{*}(2317)$ can be dynamically generated and is in the $\overline{\mathbf{3}}$. Its nonstrange partners are the lowest $D_{0}^{*}$ isospin-doublet, and their masses are about $2.1 \mathrm{GeV}[29,30,63]$, much lower than the measured value of the $D_{0}^{*}(2400)$.

The $D_{0}^{*}(2400)$ (and $D_{1}(2430)$ ) resonance parameters in the RPP [13] were obtained using the Breit-Wigner parameterization in fitting to the $D^{(*)} \pi$ invariant mass distributions. They should be questioned for two reasons: the chiral symmetry of QCD is not incorporated at the lower end of the resonance; coupled channels $\left(D^{(*)} \eta, D_{s}^{(*)} \bar{K}\right)$ are not considered at the higher end. Both factors can be taken into account in the unitarized chiral approach.

The lattice results of the scattering lengths in a few channels at several pion masses mentioned above $[45,46]$ were used to fix the parameters in the next-to-leading order 
UCHPT [46]. Using heavy quark spin and flavor symmetries [65], this leads to predictions of a family of states listed in Table 2. One sees that the masses of the $D_{s 0}^{*}$ and $D_{s 1}$ are in a remarkable agreement with the measured $D_{s 0}^{*}(2317)$ and $D_{s 1}(2460)$ masses [13]. Using the relation between the scattering length and the compositeness for shallow $S$-wave bound states [66], it is found that the $D K$ molecular component of the $D_{s 0}^{*}(2317)$ wave function is about $70 \%$. Qualitatively similar result, i.e., the $D_{s 0}^{*}(2317)$ as mainly a $D K$ molecule, was also obtained in Ref. [67] by analyzing the lattice data of Ref. [43], as well as in the lattice calculation of Ref. [44].

A very intriguing feature of the predictions in Table 2 is that there are two $D_{0}^{*}$ states, and the lower one has a mass much lower than $2.4 \mathrm{GeV}$ as mentioned above. It turns out that the lower one corresponds to the SU(3) nonstrange partners of the $D_{s 0}^{*}(2317)$, while the higher one arises from the flavor sextet, apparently beyond the conventional quark model for mesons. One may wonder whether the predictions are reliable. It was demonstrated [63] that the $I=1 / 2$ energy levels in the center-of-mass frame computed by the Hadron Spectrum Collaboration with $M_{\pi} \simeq 391 \mathrm{MeV}$ [48] can be successfully described using the same parameters fixed in [46]. ${ }^{1}$ This provides a support. Recently, using updated parameters (the moving-frame data in [48] were also included in the fit), it is found that the two-pole scenario persists [58]. The question is whether the two- $D_{0}^{*}$ scenario is consistent with experimental measurements which were well fitted using a single $D_{0}^{*}(2400)$. This is the focus of the next section.

\section{Confronting two $D_{0}^{*}$ states with the LHCb data}
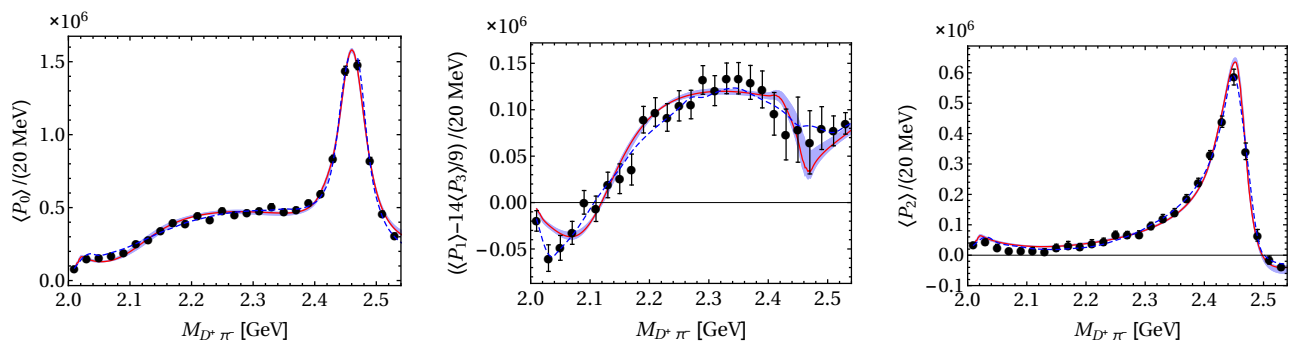

Figure 2. Fit to the LHCb data for the angular moments $\left\langle P_{0}\right\rangle,\left\langle P_{1}\right\rangle-14\left\langle P_{3}\right\rangle / 9$ and $\left\langle P_{2}\right\rangle$ for the $B^{-} \rightarrow D^{+} \pi^{-} \pi^{-}$reaction [69]. The solid lines are the best fit results, with error bands the one-sigma uncertainties propagated from the input scattering amplitudes. The dashed lines represent the LHCb fit [69]. For more details, see Ref. [57].

The best data that can be used to constrain the $D_{0}^{*}$ spectrum are from three-body $B$ decays. In particular, the $B^{-} \rightarrow D^{+} \pi^{-} \pi^{-}$has an advantage that there are no cross-channel $\pi^{-} \pi^{-}$ resonances. In fact, the first observations of the $D_{0}^{*}(2400)$ and $D_{1}(2430)$ were made in the $B^{-} \rightarrow D^{(*)+} \pi^{-} \pi^{-}$processes by Belle [32]. Recently, the LHCb experiment measured the angular moments for this process in Ref. [69]. Different angular moments are sensitive to different combinations of the partial-wave phases.

The LHCb data for the lowest few moments are fitted in Ref. [57] using a decay amplitude taking into account unitarity. The coupled-channel $\left(D \pi, D \eta, D_{s} \bar{K}\right)$ scattering amplitudes enter the decay through final state interactions. When partial waves with $L \geq 3$ are neglected,

\footnotetext{
${ }^{1}$ The description of the lattice energy levels in [44] relevant for the $D_{s 0}^{*}(2317)$ and $D_{s 1}(2460)$ using the same parameters can be found in Ref. [68].
} 
which is a good approximation for $M_{D \pi} \lesssim 2.55 \mathrm{GeV}$ as indicated by the LHCb data, one can construct a special linear combination $\left\langle P_{13}\right\rangle \equiv\left\langle P_{1}\right\rangle-\frac{14}{9}\left\langle P_{3}\right\rangle$ where the $D$-wave contribution is cancelled out. It is particularly sensitive to the $S$-wave phase motion, and thus sensitive to the $D_{0}^{*}$ spectrum in that energy region. As can be seen from Fig. 2, the data can be well described with the amplitudes having two $D_{0}^{*}$ states.

From Fig. 2, the data of $\left\langle P_{13}\right\rangle$ show a drastic variation between 2.4 and $2.5 \mathrm{GeV}$. Such a variation naturally appears as there must be two cusps at the $D^{0} \eta$ and $D_{s}^{+} K^{-}$thresholds at 2.413 and $2.462 \mathrm{GeV}$, respectively. The higher cusp gets amplified by the higher pole which couples most strongly to the $D_{s} \bar{K}$ channel.

\section{Summary and outlook}

To summarize, there have been significant progress in understanding the positive-parity charmed mesons, thanking recent developments in lattice calculations, UCHPT analyses, and precise experimental measurements. The lattice results and the relevant UCHPT analyses on the charm-strange mesons suggest that the main components of the $D_{s 0}^{*}(2317)$ and $D_{s 1}(2460)$ are $D K$ and $D^{*} K$, respectively. This solves the first mystery. In this hadronic molecule scenario, the nearly equal mass splittings in the second mystery is a natural consequence of the heavy quark spin symmetry. The resolution of the last mystery comes from that the RPP masses do not represent the lowest $D_{0}^{*}$ and $D_{1}$. There are two broad $D_{0}^{*}$ states in that region, and the lowest one has a mass smaller than that of the $D_{s 0}^{*}(2317)$. Yet, the smoking gun for the $D_{s 0}^{*}(2317)$ to be a $D K$ molecule is a width of $O(100 \mathrm{keV})[46,70]$, while other scenarios expect a much smaller value. This will be measured by the PANDA experiment. Very recently, BESIII measured the branching fraction of the $D_{s 0}^{*}(2317)^{-} \rightarrow D_{s}^{-} \pi^{0}$ to be $1.00_{-0.14}^{+0.00} \pm 0.14$ [71]. Further suggestions for Belle-II and LHCb include:

- To measure precisely the angular moments, in particular $\left\langle P_{1}\right\rangle-14\left\langle P_{3}\right\rangle / 9$, for the $B \rightarrow$ $D^{(*)} \pi \pi$ and $B \rightarrow D_{s}^{(*)} \bar{K} \pi$ reactions.

- To measure the $D_{s}^{(*)} \bar{K}$ spectrum in processes such as $B^{-} \rightarrow D_{s}^{(*)+} K^{-} \pi^{-}, B^{-} \rightarrow D_{s}^{(*)+} K^{-} K^{-}$, $\bar{B}_{s}^{0} \rightarrow D_{s}^{(*)+} \bar{K}^{0} \pi^{-}$and so on. This is because the higher $D_{0}^{*}$ and $D_{1}$ states couples strongly to the $D_{s}^{(*)} \bar{K}$ and could show up as near-threshold enhancements. There have been hints in the BaBar and Belle data of $B^{-} \rightarrow D_{s}^{+} K^{-} \pi^{+}[72,73], B^{0} \rightarrow D_{s}^{-} K_{S} \pi^{+}$and $B^{+} \rightarrow D_{s}^{-} K^{+} K^{+}$[74].

- To search for the bottom partners of the $D_{s 0}^{*}(2317)(M \simeq 5.72 \mathrm{GeV})$ in $B_{s}^{*} \gamma$ and $D_{s 1}(2460)$ $(M \simeq 5.77 \mathrm{GeV})$ in $B_{s}^{(*)} \gamma$. Notice that the latter is not the known $B_{s 1}(5830)$.

Moreover, the existence of the flavor-sextet state can be searched for on lattice using large SU(3) symmetric quark masses, and the bottom-nonstrange $0^{+}$and $1^{+}$mesons can also be studied on lattice.

It is foreseen that the nature of the positive-parity heavy mesons will be revealed with the joint efforts from experimental, lattice QCD and theoretical sides.

We are grateful to the supports received from the National Natural Science Foundation of China under Grant No. 11621131001, No. 11747601 and No. 11835015, from the Chinese Acedamy of Sciences (CAS) under Grunt No. QYZDB-SSW-SYS013 and No. XDPB09, by the CAS Center for Excellence in Particle Physics (CCEPP).

\section{References}

[1] B. Aubert et al. (BaBar), Phys. Rev. Lett. 90, 242001 (2003), hep-ex/0304021

[2] H.X. Chen, W. Chen, X. Liu, S.L. Zhu, Phys. Rept. 639, 1 (2016), 1601.02092 
[3] H.X. Chen, W. Chen, X. Liu, Y.R. Liu, S.L. Zhu, Rept. Prog. Phys. 80, 076201 (2017), 1609.08928

[4] R.F. Lebed, R.E. Mitchell, E.S. Swanson, Prog. Part. Nucl. Phys. 93, 143 (2017), 1610.04528

[5] A. Esposito, A. Pilloni, A.D. Polosa, Phys. Rept. 668, 1 (2016), 1611.07920

[6] F.K. Guo, C. Hanhart, U.G. Meißner, Q. Wang, Q. Zhao, B.S. Zou, Rev. Mod. Phys. 90, 015004 (2018), 1705.00141

[7] A. Ali, J.S. Lange, S. Stone, Prog. Part. Nucl. Phys. 97, 123 (2017), 1706.00610

[8] S.L. Olsen, T. Skwarnicki, D. Zieminska, Rev. Mod. Phys. 90, 015003 (2018), 1708.04012

[9] M. Karliner, J.L. Rosner, T. Skwarnicki, Ann. Rev. Nucl. Part. Sci. 68, 17 (2018), 1711.10626

[10] W. Altmannshofer et al. (Belle II) (2018), 1808. 10567

[11] A. Cerri et al. (2018), 1812.07638

[12] D. Besson et al. (CLEO), Phys. Rev. D68, 032002 (2003), [Erratum: Phys. Rev.D75,119908(2007)], hep-ex/0305100

[13] M. Tanabashi et al. (Particle Data Group), Phys. Rev. D98, 030001 (2018)

[14] S. Godfrey, N. Isgur, Phys. Rev. D32, 189 (1985)

[15] S. Godfrey, K. Moats, Phys. Rev. D93, 034035 (2016), 1510.08305

[16] R.N. Cahn, J.D. Jackson, Phys. Rev. D68, 037502 (2003), hep-ph/0305012

[17] S. Godfrey, Phys. Lett. B568, 254 (2003), hep-ph/0305122

[18] P. Colangelo, F. De Fazio, Phys. Lett. B570, 180 (2003), hep-ph/0305140

[19] T. Mehen, R.P. Springer, Phys. Rev. D72, 034006 (2005), hep-ph/0503134

[20] O. Lakhina, E.S. Swanson, Phys. Lett. B650, 159 (2007), hep-ph/0608011

[21] W.A. Bardeen, E.J. Eichten, C.T. Hill, Phys. Rev. D68, 054024 (2003), hep-ph/0305049

[22] M.A. Nowak, M. Rho, I. Zahed, Acta Phys. Polon. B35, 2377 (2004), hep-ph/0307102

[23] L. Maiani, F. Piccinini, A.D. Polosa, V. Riquer, Phys. Rev. D71, 014028 (2005), hep-ph/0412098

[24] T.E. Browder, S. Pakvasa, A.A. Petrov, Phys. Lett. B578, 365 (2004), hep-ph/0307054

[25] A.P. Szczepaniak, Phys. Lett. B567, 23 (2003), hep-ph/0305060

[26] T. Barnes, F.E. Close, H.J. Lipkin, Phys. Rev. D68, 054006 (2003), hep-ph/0305025

[27] E. van Beveren, G. Rupp, Phys. Rev. Lett. 91, 012003 (2003), hep-ph/0305035

[28] Y.Q. Chen, X.Q. Li, Phys. Rev. Lett. 93, 232001 (2004), hep-ph/0407062

[29] E.E. Kolomeitsev, M.F.M. Lutz, Phys. Lett. B582, 39 (2004), hep-ph/0307133

[30] F.K. Guo, P.N. Shen, H.C. Chiang, R.G. Ping, B.S. Zou, Phys. Lett. B641, 278 (2006), hep-ph/0603072

[31] F.K. Guo, P.N. Shen, H.C. Chiang, Phys. Lett. B647, 133 (2007), hep-ph/0610008

[32] K. Abe et al. (Belle), Phys. Rev. D69, 112002 (2004), hep-ex/0307021

[33] J.M. Link et al. (FOCUS), Phys. Lett. B586, 11 (2004), hep-ex/0312060

[34] B. Aubert et al. (BaBar), Phys. Rev. D79, 112004 (2009), 0901. 1291

[35] R. Aaij et al. (LHCb), Phys. Rev. D92, 012012 (2015), 1505.01505

[36] R. Aaij et al. (LHCb), Phys. Rev. D92, 032002 (2015), 1505.01710

[37] P. Colangelo, F. De Fazio, F. Giannuzzi, S. Nicotri, Phys. Rev. D86, 054024 (2012), 1207.6940

[38] M.H. Alhakami, Phys. Rev. D93, 094007 (2016), 1603.08848

[39] H.Y. Cheng, F.S. Yu, Eur. Phys. J. C77, 668 (2017), 1704.01208 
[40] G.S. Bali, Phys. Rev. D68, 071501 (2003), hep-ph/0305209

[41] A. Dougall, R.D. Kenway, C.M. Maynard, C. McNeile (UKQCD), Phys. Lett. B569, 41 (2003), hep-lat/0307001

[42] D. Mohler, C.B. Lang, L. Leskovec, S. Prelovsek, R.M. Woloshyn, Phys. Rev. Lett. 111, 222001 (2013), 1308. 3175

[43] C.B. Lang, L. Leskovec, D. Mohler, S. Prelovsek, R.M. Woloshyn, Phys. Rev. D90, 034510 (2014), 1403.8103

[44] G.S. Bali, S. Collins, A. Cox, A. Schäfer, Phys. Rev. D96, 074501 (2017), 1706.01247

[45] L. Liu, H.W. Lin, K. Orginos, PoS LATTICE2008, 112 (2008), 0810. 5412

[46] L. Liu, K. Orginos, F.K. Guo, C. Hanhart, U.G. Meißner, Phys. Rev. D87, 014508 (2013), 1208.4535

[47] D. Mohler, S. Prelovsek, R.M. Woloshyn, Phys. Rev. D87, 034501 (2013), 1208.4059

[48] G. Moir, M. Peardon, S.M. Ryan, C.E. Thomas, D.J. Wilson, JHEP 10, 011 (2016), 1607.07093

[49] J. Hofmann, M.F.M. Lutz, Nucl. Phys. A733, 142 (2004), hep-ph/0308263

[50] D. Gamermann, E. Oset, D. Strottman, M.J. Vicente Vacas, Phys. Rev. D76, 074016 (2007), hep-ph/0612179

[51] J.M. Flynn, J. Nieves, Phys. Rev. D75, 074024 (2007), hep-ph/0703047

[52] F.K. Guo, C. Hanhart, U.G. Meißner, Eur. Phys. J. A40, 171 (2009), 0901.1597

[53] P. Wang, X.G. Wang, Phys. Rev. D86, 014030 (2012), 1204.5553

[54] M. Altenbuchinger, L.S. Geng, W. Weise, Phys. Rev. D89, 014026 (2014), 1309.4743

[55] Z.H. Guo, U.G. Meißner, D.L. Yao, Phys. Rev. D92, 094008 (2015), 1507.03123

[56] M.L. Du, F.K. Guo, U.G. Meißner, D.L. Yao, Eur. Phys. J. C77, 728 (2017), 1703.10836

[57] M.L. Du, M. Albaladejo, P. Fernández-Soler, F.K. Guo, C. Hanhart, U.G. Meißner, J. Nieves, D.L. Yao, Phys. Rev. D98, 094018 (2018), 1712 . 07957

[58] Z.H. Guo, L. Liu, U.G. Meißner, J.A. Oller, A. Rusetsky, Eur. Phys. J. C79, 13 (2019), 1811.05585

[59] T.N. Truong, Phys. Rev. Lett. 61, 2526 (1988)

[60] J.A. Oller, E. Oset, Phys. Rev. D60, 074023 (1999), hep-ph/9809337

[61] J.R. Pelaez, Phys. Rept. 658, 1 (2016), 1510.00653

[62] T. Hyodo, D. Jido, A. Hosaka, Phys. Rev. D75, 034002 (2007), hep-ph/0611004

[63] M. Albaladejo, P. Fernandez-Soler, F.K. Guo, J. Nieves, Phys. Lett. B767, 465 (2017), 1610.06727

[64] C.B. Lang, D. Mohler, S. Prelovsek, R.M. Woloshyn, Phys. Lett. B750, 17 (2015), 1501.01646

[65] M. Neubert, Phys. Rept. 245, 259 (1994), hep-ph/9306320

[66] S. Weinberg, Phys. Rev. 137, B672 (1965)

[67] A. Martínez Torres, E. Oset, S. Prelovsek, A. Ramos, JHEP 05, 153 (2015), 1412 . 1706

[68] M. Albaladejo, P. Fernandez-Soler, J. Nieves, P.G. Ortega, Eur. Phys. J. C78, 722 (2018), 1805.07104

[69] R. Aaij et al. (LHCb), Phys. Rev. D94, 072001 (2016), 1608.01289

[70] M.F.M. Lutz, M. Soyeur, Nucl. Phys. A813, 14 (2008), 0710. 1545

[71] M. Ablikim et al. (BESIII), Phys. Rev. D97, 051103 (2018), 1711.08293

[72] B. Aubert et al. (BaBar), Phys. Rev. Lett. 100, 171803 (2008), 0707. 1043

[73] J. Wiechczynski et al. (Belle), Phys. Rev. D80, 052005 (2009), 0903.4956

[74] J. Wiechczynski et al. (Belle), Phys. Rev. D91, 032008 (2015), 1411.2035 